OPEN ACCESS

Edited by: Ann Horgas,

University of Florida, United States

Reviewed by:

Fernand Anton,

University of

Luxembourg, Luxembourg

Daniel Whibley

University of Michigan, United States

*Correspondence:

Karen O. Moss

moss.391@osu.edu

Specialty section: This article was submitted to

Geriatric Pain,

a section of the journal

Frontiers in Pain Research

Received: 20 September 2021 Accepted: 25 October 2021

Published: 17 November 2021

Citation

Moss KO, Wright KD, Tan A, Rose $K M$, Scharre DW, Gure TR, Cowan RL, Failla MD and Monroe TB

(2021) Race-Related Differences

Between and Within Sex to Experimental Thermal Pain in Middle and Older Adulthood: An Exploratory

Pilot Analysis.

Front. Pain Res. 2:780338. doi: 10.3389/fpain.2021.780338

\section{Race-Related Differences Between and Within Sex to Experimental Thermal Pain in Middle and Older Adulthood: An Exploratory Pilot Analysis}

\author{
Karen O. Moss ${ }^{1,2,3 *}$, Kathy D. Wright ${ }^{1,2,4}$, Alai Tan ${ }^{1}$, Karen M. Rose ${ }^{1,2}$, \\ Douglas W. Scharre ${ }^{5,6}$, Tanya R. Gure ${ }^{6,7}$, Ronald L. Cowan ${ }^{8}$, Michelle D. Failla ${ }^{1,2}$ and \\ Todd B. Monroe ${ }^{1,2}$
}

${ }^{1}$ College of Nursing, The Ohio State University, Columbus, OH, United States, ${ }^{2}$ Center for Healthy Aging, Self-Management and Complex Care, College of Nursing, The Ohio State University, Columbus, $\mathrm{OH}$, United States, ${ }^{3}$ Center for Health Outcomes in Medicine, Scholarship and Service (HOMES), College of Medicine, The Ohio State University, Columbus, OH, United States, ${ }^{4}$ Discovery Themes-Chronic Brain Injury Program, The Ohio State University, Columbus, OH, United States, ${ }^{5}$ Center for Cognitive and Memory Disorders, The Ohio State University Wexner Medical Center, Columbus, OH, United States, ${ }^{6}$ College of Medicine, The Ohio State University, Columbus, OH, United States, ${ }^{7}$ Division of General Internal Medicine and Geriatrics, The Ohio State University Wexner Medical Center, Columbus, OH, United States, ${ }^{8}$ Department of Psychiatry, College of Medicine, University of Tennessee Health Sciences Center, Memphis, TN, United States

This brief report details a pilot analysis conducted to explore racial differences in pain sensitivity and unpleasantness between cognitively healthy Black and White adults, stratified by sex. A total of 24 cognitively healthy adults (12 Black and 12 White) from two completed studies were matched by age and sex, and divided into two groups based on race. Stratified analyses by sex demonstrated that Black females reported experiencing pain intensity ratings of all three intensity sensations at lower temperatures than White females. These findings will inform future research studies to determine if these results hold true in a fully-powered sample and should include mixed methodologies, incorporating neuroimaging data to further assess this phenomenon. Improving pain assessment and management across racial/ethnic groups will help healthcare providers such as nurses and physicians to ensure optimal quality of life for all.

Keywords: experimental pain, psychophysics, race, sex, thermal pain

Pain is a complex and poorly managed international public health problem (1). According to the International Association for the Study of Pain's revised definition, pain is "an unpleasant sensory and emotional experience associated with, or resembling that associated with, actual or potential tissue damage" (2). Therefore, the pain experience is measured in multidimensional modes, including sensory (intensity) and affective (unpleasantness) components (3).

Sex-differences in pain perceptions for men and women exist in both clinical and experimental pain studies with women generally reporting greater pain sensitivity and unpleasantness relative to men $(4,5)$. When examining differences by race, Blacks report more pain compared to Whites, irrespective of age or pain type [reviewed in $(6,7)]$. A seminal study conducted by Portenoy et al. found that non-Hispanic Blacks and Hispanics reported more severe pain than Whites (8). Additionally, among nursing home residents living with cancer who consumed equivalent 
analgesics, behavioral pain reports were significantly higher among Blacks relative to Whites (9). Most literature on sex, race, and pain in adults is based upon self-report or observational techniques (10) that examine these variables separately (11) with no studies, to our knowledge, focused on the combined effects of race and sex on experimental pain.

Older Blacks, in particular, have more pain producing chronic conditions, such as arthritis, and other geriatric syndromes that are likely associated with severe pain (e.g., depressive symptoms, immobility) than Whites (12). Racial and ethnic differences also exist in pain management (7). Therefore, Blacks are at high risk for greater pain experiences that are underreported and undermanaged (7). Limited psychophysical findings thus far indicate that Blacks also experience lower pain tolerance (increased sensitivity) and higher pain ratings (more intensity) than Whites [reviewed in (6)]. Specifically, there is a need to better understand differences in central pain mechanisms and psychophysical response to pain among Blacks in order to improve their pain management and subsequently their qualityof-life (6). This information would be especially applicable for older Blacks who are at greatest risk for undermanaged pain (13). The aim of this pilot study was to explore racial differences in pain sensitivity and unpleasantness between cognitively healthy Black and White adults, stratified by sex.

\section{MATERIALS AND METHODS}

\section{Study Participants}

Participants were selected from two completed experimental pain studies (NIH/NIA K23 AG046379 and NIH/NIA R21 AG049332) using very similar psychophysical procedures and behavioral data. First, the total number of Blacks available for inclusion was 13 yet one did not have complete data leaving a total of $(n=12)$ Black adults with complete data. We then purposively selected 12 White participants matched on age and sex (see demographics in Table 1). Thus, the final sample consisted of 24 cognitively healthy adults matched by age and sex, and divided into two groups based on race.

\section{Measurements}

\section{Experimental Pain Psychophysics}

Experimental pain data were collected using the Medoc $\left.{ }^{(}\right)$QSense (14) thermal stimulus delivery system to assess the perceptions of "just noticeable," "weak/mild," and "moderate" pain intensity applied to the right thenar eminence. The baseline temperature was 30 degrees Celsius $\left({ }^{\circ} \mathrm{C}\right)$ with a ramp rate of $4^{\circ} \mathrm{C} / \mathrm{s}$. After each perception, unpleasantness scores were measured using a $0-20$ scale, with 0 indicating no pain unpleasantness and 20 indicating worst pain unpleasantness (15). A series of three trials of each perception were completed and the average temperature (intensity) ratings and unpleasantness scores recorded.

\section{Anxiety}

Trait anxiety was measured with the 20-item Spielberger Trait Anxiety Inventory (16) which focuses on anxiety proneness (20 items) with scores ranging from 20 to 80 for each subscale with higher scores indicating greater anxiety $(16,17)$.

\section{Socioeconomic Status (SES)}

The Hollingshead four-factor index of SES was used to measure SES (18). The Hollingshead SES scale measures marital, retirement, education, and occupation status (18).

\section{Body Mass Index (BMI)}

Because BMI can influence the pain experience (19), BMI was calculated on each participant to ensure there were no between group differences that might confound the results.

\section{Protocol}

This cross-sectional study occurred over 2 days in the parent studies. The protocol details can be found in Monroe et al. (20). The first day consisted of a screening and enrollment/consenting visit and the second day consisted of all other data collection procedures listed above, including psychophysics. Each visit lasted $\sim 1$ h. Participants were reimbursed $\$ 100.00 /$ day for their time. All procedures were approved by the Vanderbilt University Institutional Review Board.

\section{Statistical Analysis}

Descriptive statistics were used to summarize sample characteristics and pain ratings, by sex (female vs. male) and race (White vs. Black). In each sex stratum, differences between the racial groups were compared by: (1) means and standard deviations (SDs), (2) two-sample $t$-tests, and (3) effect sizes (Cohen's $d$ ). A $p<0.05$ was considered statistically significant. The Cohen's $d$-values of $0.2,0.5$, and 0.8 were cutoffs for small, medium, and large effect sizes, respectively. All analyses were conducted using SAS 9.4 (SAS ${ }^{\circledR}$ Institute, Cary, North Carolina).

\section{RESULTS}

Table 1 includes the sample characteristics by race and sex. Mean age for Black participants was 63.9 years (SD: 10.5) and 64.2 years (SD: 10.2) for White participants. There were no significant between-group differences in age. White females had higher BMI $(d=0.57)$, and lower trait anxiety $(d=-0.66)$ than Black females, while White males had lower BMI $(d=-0.91)$ than Black males. Stratified analyses by sex demonstrated that Black females reported experiencing pain intensity ratings of all three intensity sensations at lower temperatures than White females, especially for "weak/mild pain" (mean \pm SD: $35.0 \pm 2.2$ vs. $40.3 \pm 2.3, p=0.002(21), d=2.58)$, respectively. In contrast, Black males reported similar intensity pain ratings at all three intensity sensations as White males. There were no statistically significant differences for unpleasantness scores. However, Black participants reported higher scores for "just noticeable" pain unpleasantness and lower scores for "weak/mild" and "moderate" pain unpleasantness. These differences were of small to medium effect sizes with absolute temperature differences at 0.9-1.5 degrees (Table 1). 
TABLE 1 | Sample characteristics and racial difference in pain by sex.

\begin{tabular}{|c|c|c|c|c|c|c|c|c|c|c|c|c|}
\hline & \multicolumn{6}{|c|}{ Female } & \multicolumn{6}{|c|}{ Male } \\
\hline & \multicolumn{2}{|c|}{$\begin{array}{l}\text { White } \\
(n=6)\end{array}$} & \multicolumn{2}{|c|}{$\begin{array}{c}\text { Black } \\
(n=6)\end{array}$} & \multicolumn{2}{|c|}{ White vs. Black } & \multicolumn{2}{|c|}{$\begin{array}{l}\text { White } \\
(n=6)\end{array}$} & \multicolumn{2}{|c|}{$\begin{array}{c}\text { Black } \\
(n=6)\end{array}$} & \multicolumn{2}{|c|}{ White vs. Black } \\
\hline & Mean & SD & Mean & SD & Cohen's D & $\mathbf{P}$ & Mean & SD & Mean & SD & Cohen's D & $\mathbf{P}$ \\
\hline \multicolumn{13}{|c|}{ Sample characteristics } \\
\hline Age & 65.3 & 12.2 & 65.2 & 10.7 & 0.02 & 0.980 & 63.0 & 8.8 & 62.7 & 11.2 & 0.04 & 0.955 \\
\hline Body Mass Index & 27.2 & 5.3 & 24.0 & 6.7 & $0.57^{++}$ & 0.390 & 28.4 & 4.9 & 33.1 & 6.4 & $-0.91^{++}$ & 0.182 \\
\hline Socioeconomic Status & 48.5 & 9.7 & 43.9 & 18.1 & $0.35^{+}$ & 0.597 & 52.9 & 10.9 & 51.7 & 5.8 & 0.15 & 0.865 \\
\hline Trait Anxiety & 47.5 & 3.0 & 49.7 & 4.1 & $-0.66^{++}$ & 0.320 & 46.5 & 2.5 & 46.8 & 3.7 & -0.12 & 0.859 \\
\hline \multicolumn{13}{|c|}{ Intensity pain ratings (Medoc ${ }^{\circledR} \mathbf{Q}$-sense) } \\
\hline Just Noticeable & 33.2 & 1.5 & 31.8 & 0.4 & $1.35^{+++}$ & 0.078 & 34.7 & 1.4 & 34.7 & 1.2 & 0.00 & 1.000 \\
\hline Weak/Mild & 40.3 & 2.3 & 35.0 & 2.2 & $2.58^{+++}$ & 0.002 & 38.5 & 3.5 & 39.8 & 3.3 & $-0.43^{+}$ & 0.510 \\
\hline Moderate & 44.8 & 2.8 & 41.5 & 2.7 & $1.32^{+++}$ & 0.063 & 43.0 & 3.2 & 43.3 & 4.1 & -0.10 & 0.878 \\
\hline \multicolumn{13}{|c|}{ Unpleasantness pain scores (0-20 Scale) } \\
\hline Just Noticeable & 0.3 & 0.5 & 1.5 & 3.2 & $-0.56^{++}$ & 0.418 & 0.8 & 1.0 & 1.7 & 1.5 & $-0.72^{++}$ & 0.283 \\
\hline Weak/Mild & 3.3 & 2.3 & 2.5 & 2.6 & $0.38^{+}$ & 0.565 & 5.5 & 0.8 & 4.2 & 2.8 & $0.71^{++}$ & 0.305 \\
\hline Moderate & 8.3 & 2.3 & 7.3 & 3.8 & $0.35^{+}$ & 0.597 & 9.5 & 1.4 & 8.0 & 5.0 & $0.45^{+}$ & 0.505 \\
\hline
\end{tabular}

Effect size: ${ }^{+}$Small $(0.2 \leq$ Cohen's $d<0.5) ;{ }^{++}$Medium $(0.5 \leq d<0.8) ;{ }^{+++}$Large $(d \geq 0.8)$.

\section{DISCUSSION}

Though a relatively small sample, the current exploratory pilot results support and extend previously conducted research on race, sex, and pain. Established literature indicates that females are generally more sensitive to pain, have more pain related diagnoses, and report pain as more intense (5). However, the focus of most research on sex differences in pain does not include race as a primary predictor variable and similarly most research on racial differences in pain does not include within sex as a group comparison $(5,7)$.

Current pilot results support the notion that the interaction of race and sex may have important effects when designing future studies and preparing clinical practice guidelines. Overall, the current study demonstrated patterns of experimental pain responses, similar to previous research $(6,7)$. Similar to overall findings by race from Portenoy et al., when subdivided by sex in the current study, Black females were more sensitive to pain than White females (21). Although preliminary, the relationship between pain differences among Black and White females was robust. However, our sample size did not have sufficient power to detect statistically significant associations for effect size that are smaller than 1.8. For example, the racial differences of just noticeable unpleasantness pain scores were of medium to large effect sizes for both females and males ( $d=$ -0.56 and -0.72 , respectively), even though the differences did not reach statistical significance ( $p=0.42$ and 0.28 , respectively). Therefore, we reported both effect sizes and statistical $p$-values to guide results interpretation. Because of the small size we did not adjust for demographic and behavioral covariates (e.g., Age, SES, BMI, anxiety).

These pilot findings will inform future research that should seek to determine whether these findings are supported in a larger study including multiple psychophysical tests (e.g., threshold, tolerance, temporal summation, conditioned pain modulation) and multiple experimental pain measures (e.g., thermal, mechanical pressure, cold pressure, electrical shock). In addition to added experimental psychophysical procedures, more studies are needed to determine the impact and presence of geriatric co-morbidities that affect pain perception and intensity, which may differ by race and sex (e.g., cancer, diabetes, osteoarthritis). Thus, additional experimental and clinical studies of pain are needed in larger samples to provide more evidence to inform assessment and treatment of pain in racially diverse groups of male and female adults.

Because this study included mostly older adults and were purposefully age-matched, generalizability of findings is limited. To improve our understanding of the pain experience and pain mechanisms in this population, future studies using neuroimaging and mixed-methods approaches are needed.

Assessment and management of pain can be optimized when healthcare providers, such as nurses and physicians are able to better understand pain intensity and unpleasantness differences in adults from diverse sex and racial backgrounds. This is a necessary first step to ensure optimal quality of life through improvements in pain management for all adults, regardless of their sex or race.

\section{DATA AVAILABILITY STATEMENT}

The datasets used and/or analyzed during the current study are available from the senior author on reasonable request. Requests to access these datasets should be directed to Todd Monroe (monroe.1181@osu.edu). 


\section{ETHICS STATEMENT}

The studies involving human participants were reviewed and approved by Vanderbilt University Institutional Review Board. The patients/participants provided their written informed consent to participate in this study.

\section{AUTHOR CONTRIBUTIONS}

$\mathrm{KM}$ : investigation, writing-original draft, writing-review and editing, visualization, and project administration. KW, KR, DS, TG, and MF: writing-review and editing and visualization. AT: formal analysis, resources, data curation, writing-review and

\section{REFERENCES}

1. Husebo BS, Achterberg W, Flo E. Identifying and managing pain in people with Alzheimer's disease and other types of dementia: a systematic review. CNS Drugs. (2016) 30:481-97. doi: 10.1007/s40263-0160342-7

2. Raja SN, Carr DB, Cohen M, Finnerup NB, Flor H, Gibson S, et al. The revised International Association for the Study of Pain definition of pain: Concepts, challenges, and compromises. Pain. (2020) 161:197682. doi: 10.1097/j.pain.0000000000001939

3. Petzke F, Harris RE, Williams DA, Clauw DJ, Gracely RH. Differences in unpleasantness induced by experimental pressure pain between patients with fibromyalgia and healthy controls. Eur J Pain. (2005) 9:32535. doi: 10.1016/j.ejpain.2004.09.001

4. Bartley EJ, Fillingim RB. Sex differences in pain: a brief review of clinical and experimental findings. Br J Anaesth. (2013) 111:52-8. doi: 10.1093/bja/aet127

5. Fillingim RB, King CD, Ribeiro-Dasilva MC, Rahim-Williams B, Riley JL. Sex, gender, and pain: A review of recent clinical and experimental findings. J Pain. (2009) 10:447-85. doi: 10.1016/j.jpain.2008.12.001

6. Kim HJ, Yang GS, Greenspan JD, Downton KD, Griffith KA, Renn CL, et al. Racial and ethnic differences in experimental pain sensitivity: systematic review and meta-analysis. Pain. (2017) 158:194-211. doi: 10.1097/j.pain.0000000000000731

7. Green CR, Anderson KO, Baker TA, Campbell LC, Decker S, Fillingim RB, et al. The unequal burden of pain: confronting racial and ethnic disparities in pain. Pain Med. (2003) 4:277-94. doi: 10.1046/j.1526-4637.2003.03034.x

8. Portenoy RK, Ugarte C, Fuller I, Haas G. Population-based survey of pain in the United States: differences among White, African American, and Hispanic subjects. J Pain. (2004) 5:317-28. doi: 10.1016/j.jpain.2004.05.005

9. Monroe TB, Carter MA. A retrospective pilot study of African-American and Caucasian nursing home residents with dementia who died from cancer. J Pain Symptom Manage. (2010) 40:e1. doi: 10.1016/j.jpainsymman.2010. 06.007

10. Hoteit F, Feldman DE, Pollice J, Carlesso LC. Scoping review of pain and patient characteristics and physical function associated with intermittent and constant pain in people with knee osteoarthritis. Physiother Canada. (2020) 73:118-28. doi: 10.3138/ptc-2019-0049

11. Ostrom C, Bair E, Maixner W, Dubner R, Fillingim RB, Ohrbach R, et al. Demographic predictors of pain sensitivity: results from the OPPERA study. $J$ Pain. (2017) 18:295-307. doi: 10.1016/j.jpain.2016.10.018

12. Koroukian SM, Schiltz NK, Warner DF, Sun J, Stange KC, Given CW, et al. Multimorbidity: constellations of conditions across subgroups of midlife and older individuals, and related Medicare expenditures. J Comorbidity. (2017) 7:33-43. doi: 10.15256/joc.2017.7.91 editing, and visualization. RC: conceptualization, methodology, validation, writing-review and editing, visualization, and funding acquisition. TM: conceptualization, methodology, validation, investigation, resources, writing-original draft, visualization, supervision, project administration, and funding acquisition. All authors contributed to the article and approved the submitted version.

\section{FUNDING}

Data accessed for this project were combined from studies supported by the National Institutes of Health, National Institute on Aging projects K23 AG046379 and R21 AG049332.

13. Booker SQ. African Americans' perceptions of pain and pain management: a systematic review. J Transcult Nurs. (2014) 27:73-80. doi: 10.1177/1043659614526250

14. Medoc. Medoc Advanced Medical Systems. (2020). Available online at: https:// www.medoc-web.com/pain-research (accessed March 19, 2021).

15. Cole LJ, Farrell MJ, Duff EP, Barber JB, Egan GF, Gibson SJ. Pain sensitivity and fMRI pain-related brain activity in Alzheimer's disease. Brain. (2006) 129:2957-65. doi: 10.1093/brain/awl228 \%J Brain

16. Spielberger, CD, Gorsuch, RL, Lushene, RE. Manual for the State-Trait Anxiety Inventory. Polo Alto: Consulting Psychologists Press (1970).

17. Jillian LJ. Measures of anxiety. Arthritis Care Res. (2011) 63:20561. doi: 10.1002/acr.20561

18. Hollingshead A. Four Factor Index of Social Status. New Haven, CT: Yale University (1975).

19. Koçyigit BF, Okyay RA. The relationship between body mass index and pain, disease activity, depression and anxiety in women with fibromyalgia. PeerJ. (2018) 6:e4917. doi: 10.7717/peerj.4917

20. Monroe TB, Gibson SJ, Bruehl SP, Gore JC, Dietrich MS, Newhouse P, et al. Contact heat sensitivity and reports of unpleasantness in communicative people with mild to moderate cognitive impairment in Alzheimer's disease: a crosssectional study. BMC Med. (2016) 14:74. doi: 10.1186/s12916-016-0619-1

21. Moss K, Atalia S, Iversen L, Rose K, Tan A, Wright K, et al. R2. Diverse perceptions of experimental thermal pain: race-related differences between and within sex. Pain Manag Nurs. (2021) 22:240. doi: 10.1016/j.pmn.2021.02.051

Conflict of Interest: The authors declare that the research was conducted in the absence of any commercial or financial relationships that could be construed as a potential conflict of interest.

Publisher's Note: All claims expressed in this article are solely those of the authors and do not necessarily represent those of their affiliated organizations, or those of the publisher, the editors and the reviewers. Any product that may be evaluated in this article, or claim that may be made by its manufacturer, is not guaranteed or endorsed by the publisher.

Copyright (c) 2021 Moss, Wright, Tan, Rose, Scharre, Gure, Cowan, Failla and Monroe. This is an open-access article distributed under the terms of the Creative Commons Attribution License (CC BY). The use, distribution or reproduction in other forums is permitted, provided the original author(s) and the copyright owner(s) are credited and that the original publication in this journal is cited, in accordance with accepted academic practice. No use, distribution or reproduction is permitted which does not comply with these terms. 